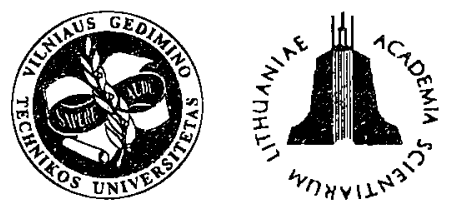

ISSN 1648-4142 TRANSPORT

http:/www.vtu.lt/english/editions

\title{
THE EVALUATION OF IMPLEMENTATION IMPACT OF CENTRALIZED TRAFFIC CONTROL SYSTEMS IN RAILWAYS
}

\author{
Tomas Magyla \\ Kaunas University of Technology.E-mail: Tomas.Magyla@eaf.ktu.lt \\ Received 200202 25; accepted 20020418
}

\begin{abstract}
In this article we propose to evaluate the utility of implementation of Centralized Traffic Control System EbiScreen using expert evaluations in indistinct numbers, in the form of trapezoid membership function, as we are confident in the advantages obtained using this evaluation method. The methodology of evaluating and weightng of criteria in a trapezoid membership form is proposed in this article together with the results of evaluation. The idea is to integrate the hicrarchy of objectives with the multi-attribute weight vectors using the values obtained by the expert group in evaluation process. The additional weights for the attributes in the multi-attribute analysis are provided within the same scale, so that they can be combined to find the overall value of the evaluation. Methodological presentation is followed by the results of sample application in Lithuanian Railways where the object of evaluation was the EbiScreen Centralized Traffic Control System.
\end{abstract}

Keywords: centralized traffic control, expert ranking, fuzzy integral, trapezoid membership.

\section{Introduction}

The modernization of Lithuanian railways infrastructure is in progress. The old relay-based manual or semiautomatic traffic control systems will be modemized changing end-of life relay systems by newly developed electronic intelligent control systems, where country-dependant mathematical train traffic routing interdependence rules will be pre - programmed so, that the complete remote control of the traffic in distant stations will be normal from the traffic control center and fully automatic routing will be possible. It means maximum throughput capacity, optimal time tables, comfortable traffic data filtering and analysis, minimum human supervision, therefore minimum expenses for maintenance and so on. All the possible benefits taken together minus the price of the system project, permission for construction work, manufacturing, installation, commissioning, maintenance costs and the possible shortcomings of the new system give the understanding of the system implementation overall impact. A new comprehensive system does not always mean a better one, if all of the possible inward effects taken together are equal or even compensate the positive side of the implementation issues. For instance [1], after the detailed analysis applying mathematical modeling and expert evaluations to a railway auto-block system, it turned out that after the implementation of a modem auto-block system instead of the existing semi-automatic block system, the new system will be able to handle traffic throughput capacity approx. one and a half times higher than the old one. This is an impressive indicator, of course. The same research included traffic growth prognosis, which claimed, that at the moment of research the old semi-automatic block system was able to handle at least three times higher throughput capacity, compared with the statistics of that time, because of the low growth of the state economy. The state railway growth prognosis was planning that this maximum capacity will only be reached in a decade or so, and it was a fact, that speaking in monetary terms, the implementation of a new automated-block system was not reasonable. That's why we need to have the method for evaluating the overall impact of a process or innovation.

So far no comprehensive approach exists for evaluating and finding objective overall impact of the implementation of a new railway projects among the most complicated decision/evaluation problems that exist. In complicated railway decision-making situations, where research objects are huge traffic interlocking (TI) or centralized traffic control systems (CTC), the evaluation and optimization based on classical methods, is impossible, as the only one acceptable way for the identification of evaluation facets is using fuzzy identification methods [2-6]. These methods are based on expert evaluations, that is, the human intellect is used as a measuring instrument. The initial phase of evaluation, when experts submit their indistinct evaluations, is of the highest importance $[7,8]$ and often determines the error of the whole evaluation result. The initial evaluation data are usually compiled in a matrix or array, containing indistinct numbers in the form of a triangle membership function [9], so that the following evaluation phase- processing of compiled data - would be easy and efficient. The processing can be based on a fuzzy 
analytic hierarchy pair-wise comparison method [10-12], weight coefficient space transformation methods [13], or other robust methods of analysis [ $14-16]$; some of inference methods may be based on the fuzzy inference software packages.

In this article we propose to evaluate the utility of the implementation of Centralized Traffic Control System EbiScreen using expert evaluations in indistinct numbers, in a form of trapezoid membership function, as we are confident in the advantages obtained using this evaluation method. The methodology of evaluating and weighting of criteria in a trapezoid membership form is proposed in this article together with the results of evaluation. The idea is to integrate the hierarchy of objectives with the multiattribute weight vectors using the values obtained by the expert group evaluation process. The additional weights for the attributes in the multi-attribute analysis are provided within the same scale, so that they can be combined to find the overall value of the evaluation. The methodological presentation is followed by the results of sample application in Lithuanian Railways where the object of evaluation was the Ebiscreen Centralized Traffic Control System.

\section{Factors of Evaluation}

The expansion and innovation process of railway traffic control systems results in many positive technical issues: better administrative, organizational and technical performance of railways, reliability, technical interaction, lower capital, operating and maintenance costs, ease of implementation and maintenance, many useful new features of a network and integration performance, psychological impression to the end user. In different performance evaluation methodologies these positive issues are taken for granted and usually only different alternatives of positive impacts are being analyzed. But the implementation of a new traffic control system also has an inward-effect: environmental impact, job loses, and other derived social shortcomings.

Environmental issues of railways electronics have long been considered as positive and environmentalfriendly, therefore no effective measures have been taken to prevent waste growth, however, there is an increasing recognition that many processes used to produce electronic systems do have environmental consequences (as a result of materials used, power consumed, or end-of life product consideration); some electronic products do have increasing disposal problems [17]. The replacement of old relay-based circuits by newly developed electronic computerized traffic control systems will result in a huge amount of electronic waste designated for landfill. An effective, decision-based recycling is one option that may aid in reducing the volume of electronic waste designated for landfill disposal, with the possible decision model, which can be motivated using expert evaluations.
Job loses are related with the declining of human supervision demand in new electronic traffic control systems. As experience shows, electronics and computer technology boost with exponential acceleration and a new Artificial Intellect mindset will be the engine for inference. Human supervision demand in the wide area Centralized Traffic Control Systems [7] is declining and needful only for occasional system maintenance procedures, but not for daily influence. That results in job loses and in turn, job loses result in many unwanted social sideband effects.

The abovementioned problems are quite important in railway transport. The proper measures can compensate or even eliminate unwanted effects, if applied to a planned situation. The situation can be planned referring the prognosis of evaluation. Our aim in this article is to propose the methodology that is capable to adequately identify the relationships between attributes and objectives of both technical and institutional issues of implementation CTC systems in railways.

\section{The Methodology of Evaluation}

The ordinary way of submitting multi-criteria evaluation of the research object (CTC) is the use of expressions in indistinct numbers with a "triangle" [18-20] type membership function.

The membership function is derived from the expert evaluation information:

1. Probability $p$, which is usually in a form of interval $(c, d)$, where the value of the evaluated criterion $A$ may be;

2 . Typical and most expected value of evaluated criterion, by the opinion of an expert (Fig 1). In this case the evaluation of a sub-target $A$ is:

$$
h_{A}=\left(\left(x_{x}\right), p\right) \text {. }
$$

This form is quite desirable for the knowledge of an engineer, but psychologically inconvenient for an expert: it may be hard to interpret separate parts of the dependence function. From psychological point of view a more convenient way is where an expert is asked to point out:

1. Interval $\left(x_{1}, x_{2}\right)$, where the most typical value of the criterion $A$ is by the opinion of an expert (see Fig 2);

2. Subjective probability $p$ (in a form of interval), under which the expert is right.

In this case the evaluation of the indication $A$ is:

$h_{A}=\left(\left(x_{1}, x_{2}\right), p\right)$.

Evaluation equation (2) can be changed with a trapezoid type membership function (see Fig 3.):

$$
\mu_{A}(x)=\left\{\begin{array}{cc}
1-\frac{x_{1}}{b}+\frac{1}{b} x, & x_{1}-b \leq x \leq x_{1} \\
1, & x_{1}<x<x_{2} \\
1+\frac{x_{2}}{b}-\frac{1}{b} x, & x_{2} \leq x \leq x_{2}+b .
\end{array}\right.
$$


where

$$
b=\left(x_{2}-x_{1}\right)^{1-p}=\frac{a(1-p)}{p} .
$$

We can easily notice that formula (3) is valid only if:

$$
x_{1}-b \geq x_{\text {min }}, x_{2}+b \leq x_{\text {max }} \text {. }
$$

Therefore for the ease of calculations usually $x_{\min }=$ $0, x_{\text {max }}=1$; or else formula (3) has to be modified.

As we can clearly see from Fig 2 and Fig 3, the evaluations in trapezoid membership functions possess almost the same functionality like the evaluations in a triangle membership functions, if only $x_{1}=x_{2}=x$ and $x_{1}^{\prime}=x_{2}^{\prime}=x^{\prime}$. If $x_{1} \neq x_{2},\left(\right.$ or $\left.x_{1}^{\prime} \neq x_{2}^{\prime}\right)$ an error appears. If $x_{1} \cong x_{2}$ is acceptable, an error may be regarded as acceptable. Therefore a correction procedure of an error is needful in this case and generally speaking, after this correction procedure methods of the triangle data processing can be applied to a trapezoid type initial evaluation data.

Let us assume that expert evaluations are of real-life, and inadequaleness which is dependent upon the [7] nonlinearity of expert's subjective efficiency function is present together with other expert shortages. Non-linearity of expert's subjective efficiency function is characteristic for non-typical decisions, projects, reforms, where expert conservatism takes place; also in making decisions where an expert is a concerned person. Trying to diminish or eliminate the mistakes derived from non-linearity of the expert's subjective efficiency function, a correction procedure is needful [7]: expert evaluations $h_{j A}(x)$ and $h^{\prime}{ }_{j A}(x)$ have to be enlarged by the number $\Delta_{j A}(x)$, equal to the $j$ th expert risk (evaluating object, process or action $A$ ) supplement function, to become "complete". The "incompleteness" elimination procedure for evaluation $h_{j A}(x)$ and $h^{\prime}{ }_{j A}(x)$, can be formulated as follows [7]:

$$
\begin{aligned}
& \left\{h^{\prime^{*}}{ }_{j A}(x),\left.h_{j A}^{*}(x)\right|_{j=\overline{1, k}}\right\}= \\
= & \left\{h^{\prime}{ }_{j A}(x)+\Delta_{j A}(x), h_{j A}(x)+\left.\Delta_{j A}(x)\right|_{j=\overline{1, k}}\right\} .
\end{aligned}
$$

Actually, the expert risk supplement function is calculated only after finding out the expert's efficiency function. This function depends on $A$ and can be time dependant. Due to this reason efficiency function has to be measured at the time of performing expert interviewing. The procedure of measuring efficiency function is quite complicated, therefore it is purposeful to measure it at single point and only then (with acceptable small error) expert's turn for non-risk can be considered constant: $\Delta_{j A}(x) \cong \Delta_{j A}$. The expert's $\Delta_{j A}$ can also be calculated using classical lottery methods [7].

Let us consider that the non-linearity of expert's subjective efficiency function has been foreseen and compensation is included into the initial evaluation phase. The issue of the shortage of expert's experience will be discussed later.
The complex indistinct evaluation [7] of an object, process or action $A$ with a triangle membership function is characterized by:

$$
H_{A}(x)=\frac{1}{\sum_{j=1}^{k} \alpha_{j}} \sum_{j=1}^{k} \alpha_{j} h_{j A}(x)
$$

where: $h_{j A}(x)$ - direct evaluation of evaluation object $A$ by the $j$-th expert; $j=1, k$ - index of the expert (the index of the goal in multi-attribute evaluations is $i=1, n) ; \alpha_{j}$ - the complex expert weight coefficient.

$$
\alpha_{j}=\beta_{j} \gamma_{j}
$$

where $j=1, k$;

$\beta_{j}$ is a weight coefficient, characterizing expert's experience level ("ability to evaluate in chime'); $\gamma_{j}$ is a weight coefficient, characterizing expert's distinctness of evaluations. For the triangle membership case the best illustration of $\beta_{j}$ is from geometrical point of view-it is a magnitude of both triangles $c x d$ and $c^{\prime} x^{\prime} d$ ' intersection point $k$ (see Fig 1). Triangle $c x d$ represents direct evaluation $h_{j A}(x)$ of object $A$ and $c^{\prime} x{ }^{\prime} d$ ' represents indirect (summarized) evaluation $h^{\prime}{ }_{j A}(x)$ of object $A$. The coefficient $\gamma_{j}$ is found as the rate of the area of intersection of these triangles:

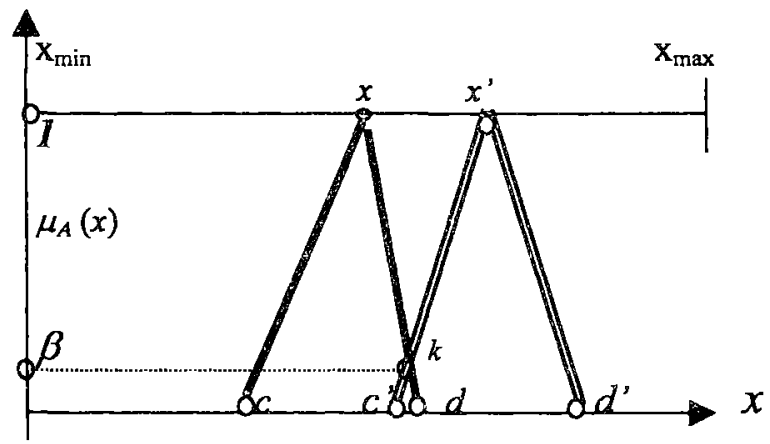

Fig 1. Evaluations submitted in a triangle type membership function

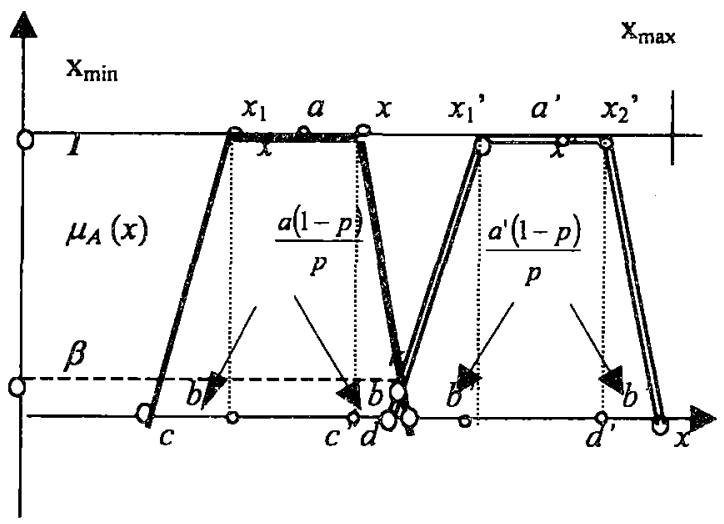

Fig 2. Evaluations submitted in a trapezoid type membership function 


$$
\gamma_{j}=c x d \cap c^{\prime} x^{\prime} d^{\prime}
$$

The bigger is the area, the more distinct is expert evaluation. The same idea can be used for the trapezoid membership case (see Fig 2). From geometrical point of view, $\beta_{j}$ is a magnitude of an intersection point $k$ of trapezoids $c x_{1} x_{2} d$ and $c^{\prime} x_{1} x_{2} x^{\prime} d$ '. Trapezoid $c x_{1} x_{2} d$ represents direct evaluation $h_{j A}(x)$ of object $A$ and a trapezoid $c x_{1}^{\prime} x_{2} d$ ' represents indirect (summarized) evaluation $h^{\prime}{ }_{j A}(x)$ of object $A$. The coefficient $\gamma_{j}$ is found as the rate of the area of intersection of these trapezoids:

$$
\gamma_{j}=c x_{1} x_{2} d \cap c^{\prime} x_{1}^{\prime} x_{2}^{\prime} d^{\prime}
$$

The bigger is the area of intersection, the more distinct is expert evaluation. In this case we would like to propose to calculate weight coefficient, characterizing expert's experience level applying fuzzy integral expres$\operatorname{sion}[7,21]$ :

$$
\beta_{j}=\frac{2 \oint_{X}\left(h_{j A}(x) \wedge h_{j A}{ }^{\prime}(x)\right) \circ x}{\int_{X} h_{j A}(x) \circ g+\int_{X} h_{j A}{ }^{\prime}(x) \circ x} ;
$$

where: $\int_{X} h_{j A}(x) \circ x$ fuzzy integral [22] of $h_{j A}(x)$ by indistinct measure $x($.) (" $\wedge$ " means "minimum");

$h_{j A}(x)$ and $h_{j A}{ }^{\prime}(x)$-indistinct evaluations, characterized by their membership functions, correspondingly $\mu(x)$ and $\mu^{\prime}(x)$; where $h_{j A}(x)$ represents (trapezoid $c x_{1} x_{2} d$ ) direct evaluation of $A$, and $h_{j A}(x)$ represents (trapezoid $c^{\prime} x_{1}$ $\left.x_{2} d^{\prime}\right)$ indirect summarized evaluation of $A$.

A weight coefficient, characterizing expert's distinctness of evaluations $\gamma_{j}$ [7] is needful for correct prognosis:

$$
\gamma_{j}=\frac{\min _{j}\left(\oint_{X} h_{j A}(x) \circ x+\oint_{X} h_{j A}{ }^{\prime}(x) \circ x\right)}{\oint_{X} h_{j A}(x) \circ x+\oint_{X} h_{j A}{ }^{\prime}(x) \circ x} .
$$

Finally, a coefficient is needful (let us call it $\varphi_{j}$ ) to correct the transformation "triangle to trapezoid" influence. This influence is important only for the trapezoid top $a$, namely for the interval $\left[x_{1}, x_{2}\right]$. If we split this interval into two equal parts $\mathrm{a} / 2=x_{1} x$ and $\mathrm{a} / 2=x x_{2}$, the central point $x$ can be treated like a median value of the triangle, the transformation error instantly becomes reduced twice, and if we include a special rated coefficient $\varphi_{j}$ :

$$
\varphi_{j}=\frac{\min _{j}\left(a_{j}\right)}{a_{j}} ; j=1, k
$$

where $\overline{a_{j}}$ is a minimal average length of trapezoid top $a_{j}$ from submitted expert evaluations. Then the complex weight coefficient for a trapezoid membership function is as follows:

$$
\theta_{j}=\beta_{j} \gamma_{j} \varphi_{j}
$$

Then the complex indistinct evaluation of an object, process or action $A$ with a trapezoid membership function is characterized by:

$$
H_{A}(x)=\frac{1}{\sum_{j=1}^{k} \theta_{j}} \sum_{j=1}^{k} \theta_{j} h_{j A}(x) .
$$

\section{Evaluation Results}

1. The technical and institutional attributes of Ebilock 950 have been experimentally discussed earlier [10], and it can be taken as an example of attribute hierarchy. For the evaluation of CTC EbiScreen we can decide on three main branches of evaluation attributes: technical, environmental and social attribute branches (Fig 3). The process of evaluation of CTC EbiScreen has included evaluation of the overall impact of CTC implementation as a sum impact value of the main three attribute branches versus the utility of the existing old CTC system. Expert submitted evaluations have been processed applying formula (15).

2. The branch of technical attributes has to be scaled down to a lower hierarchy facets: implementation issues, system costs, system performance, technical benefits and technology interaction for the end user, systern maintainability, maintenance cost and ease, technical support, administrative issues, future expansion prospects and other facets scaled down to a lower branch of hierarchy (see Fig 3).

The branch of environmental attributes: amount of waste for landfill (slag, ash, non-recyclable materials), recycling process impacts (global warming, acidification, photochemical ozone creation, possible photosmog, eutrophication), recovery rate and cost of the recycled materials as a function of recycling scenario $(\mathrm{Ag}, \mathrm{Al}, \mathrm{Cu}$, $\mathrm{Fe}, \mathrm{Hg}, \mathrm{Pb}$ and others). Recovery rate is mostly dependent on the amount of recovered metals and on the cost of recycling [17]. Fig 4 represents a wide range of railway relay equipment scrap metal composition which was found during the analysis of the environmental impact. Different scenarios of recycling have been analyzed during the evaluation of environmental issue. Due to the limitations of this article scope this evaluation results have been reviewed quite shortly.

Fig 5 represents evaluation results of environmental impact.

The branch of social impacts was evaluated as: possible unemployment rate (gross national product, societal behavior issues). This is the most indefinite issue of evaluation. A separate factor hierarchy as well as methodology can be developed for this evaluation; but in this research it was analyzed as a single factor with negative weight.

Finally, the overall value of implementation of a new CTC system is illustrated in Fig 6, as a compromise be- 


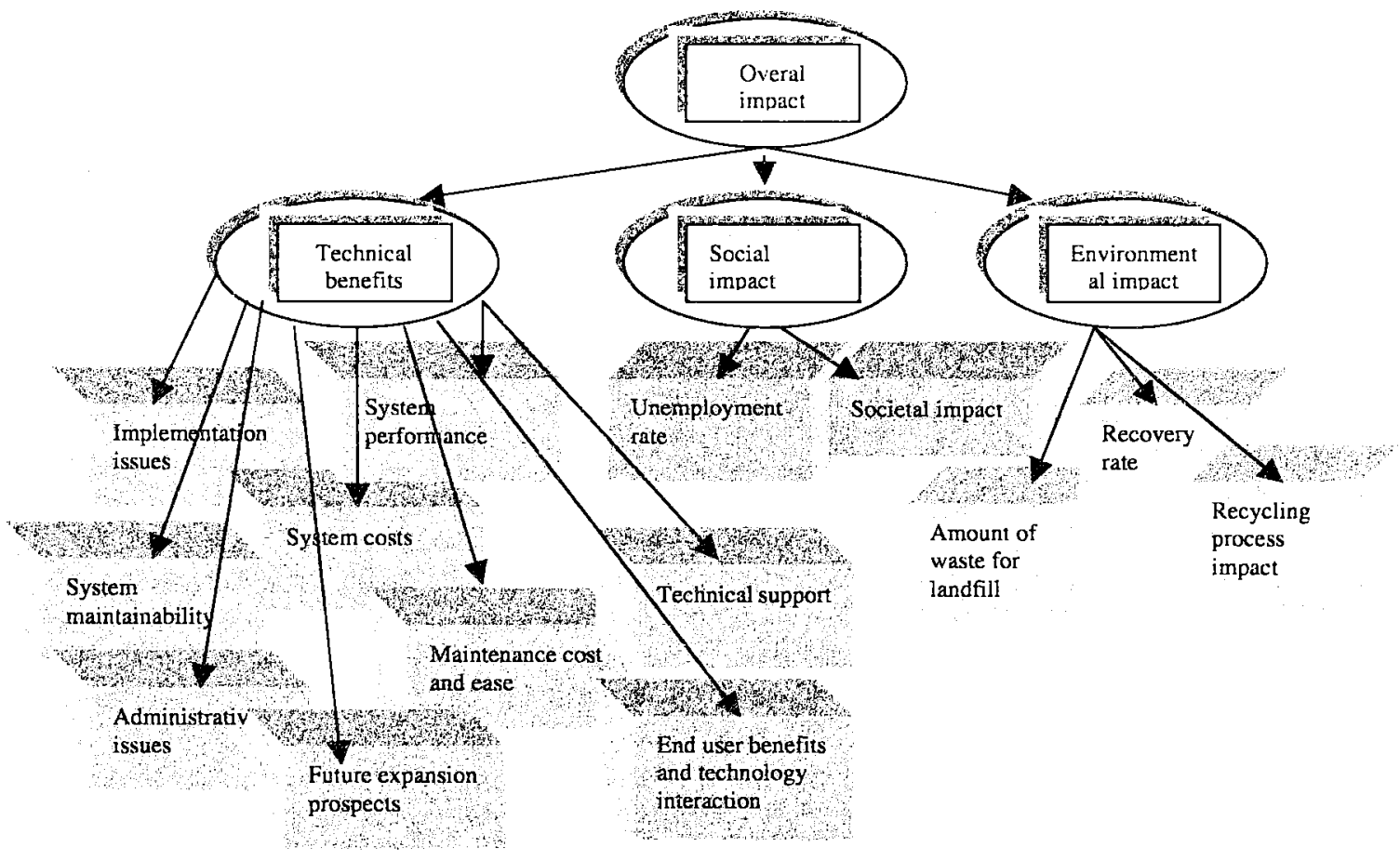

Fig 3. The main branches of evaluation attributes

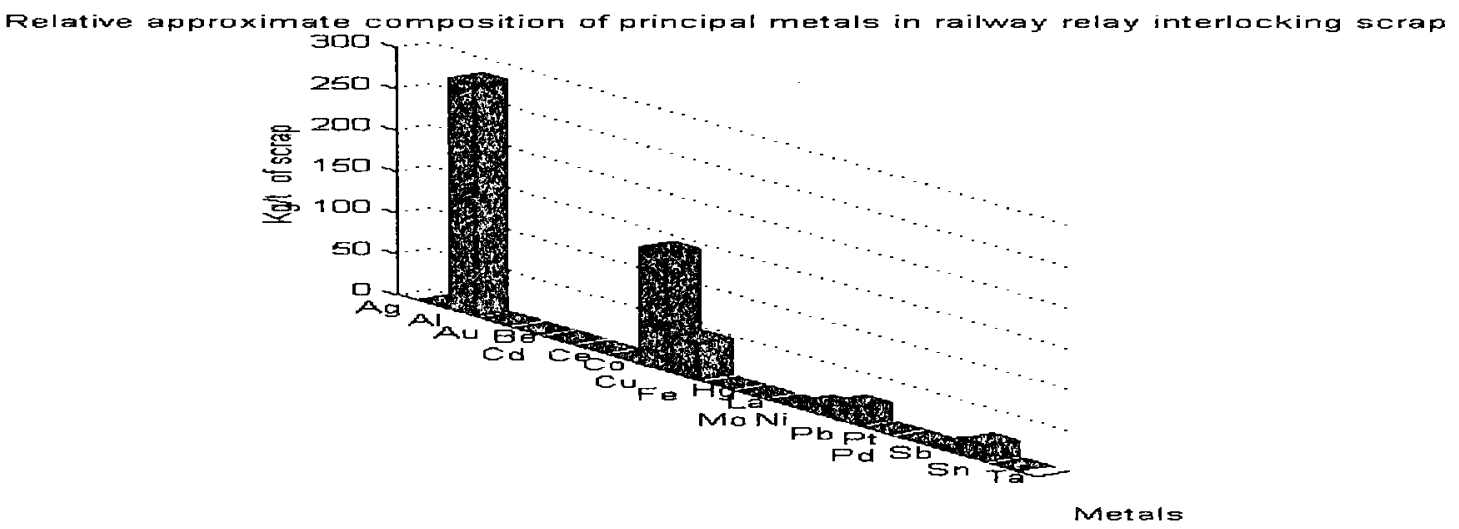

Fig 4. Composition of relay equipment scrap: metals

tween technical benefits and environmental factor together with social issues.

\section{Conclusions}

1. The overall impact or overall value of a railways traffic management system implementation is usually understood as the composition value of evaluation facets or optimal alternative versus the existing system or other alternatives, to the opinion of experts performing evaluation.

2. The widely used expert evaluation triangle membership function can be easily transformed into the evaluation with normal trapezoid membership fuzzy expression, so that the median value usage and trapezoid top splitting permit to reduce the transformation error twice.

3. A trapezoid membership function offers benefits in statistics because of the likeness of the function shape related to the Normal distribution.

4. The Inadequateness of evaluation originated because of the influence of personal interest of the expert can be compensated applying the methods of experiment planning. The expert efficiency function is time dependant and due to this reason it has to be measured at the time of perforning expert interviewing.

5. The Inadequateness of evaluations caused by the problem of external inconsistence can be compensated applying ranking to the experts performing evaluations. 


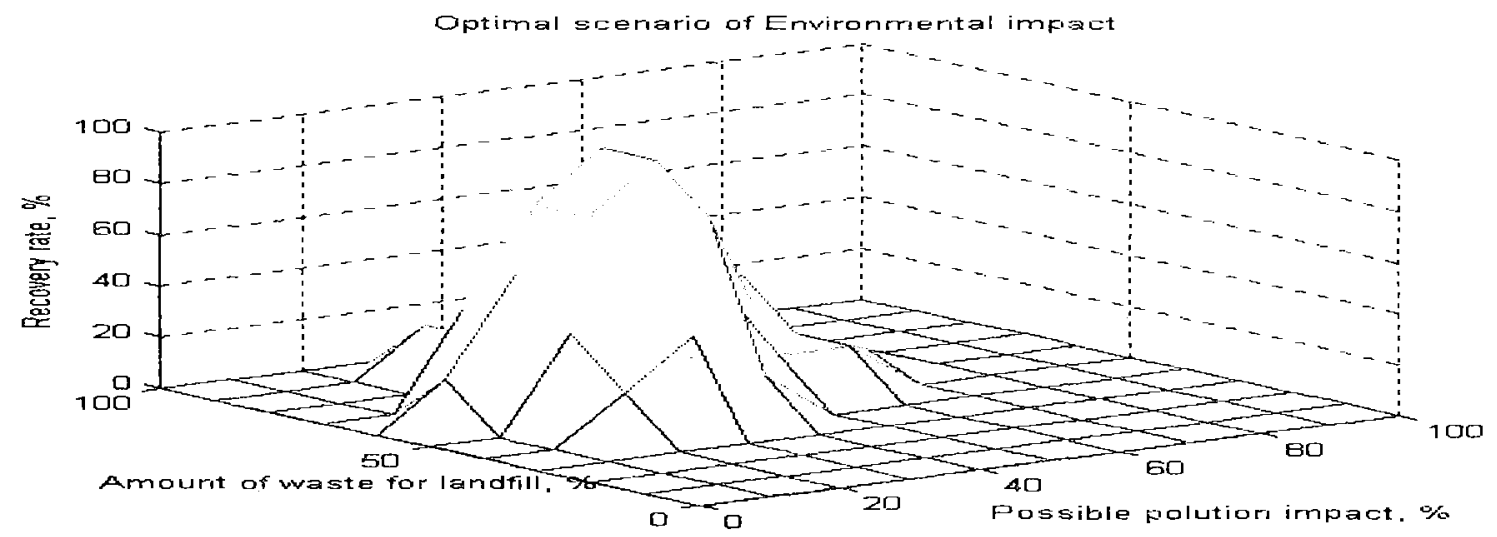

Fig 5. Expert estimation of the environmental impact: maximum recovery of precious metals and other materials at minimum cost versus minimum pollution and amount of waste for landfill

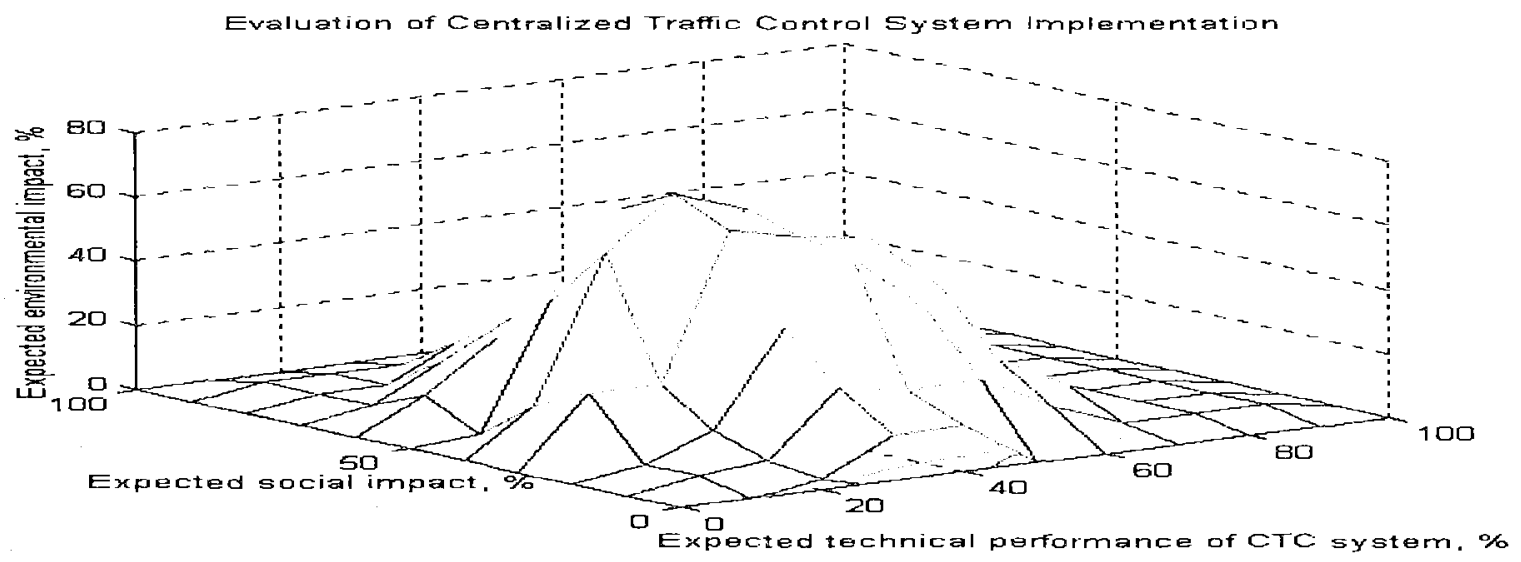

Fig 6. The overall value of the implementation impact of a new CTC, as dependence of technical, environmental and social impacts, by the opinion of experts

The additional ranking means forming complex weight coefficients for the evaluation of each expert.

6. This research has shown that the new CTC system does not always assure 100 percent perfect solution; on the contrary, it may develop new environmental or social problems. Only good planning and analysis together with the evaluation of alternatives may help.

\section{References}

1. Magyla T., Stadalius R. Research of auto-block system efficiency. In: Proceedings of international scientific conference. "Electronics-2000". Kaunas: Technology, 2000, p 87-88 (in Lithuanian).

2. Dubois D., Land J., Prade H. Automated reasoning using probabilistic logic-semantics, belief revision, and variable certainty weights. IEEE transactions on knowledge and data engineering, 1994, Vol 6, p 64-71

3. Bagdonas V., Raila M. Application of Pragmatic Criteria to the Information Systems Structure. Information Technology and Control. Kaunas: Technology, 2001, No 1 (18), p 6065.

4. Carnahan J. V., Thurston D. L., Liu T. Fuzzing ratings for multi-attribute design decision-making. Joumal of mechanical design. 1994, Vol 116 (2), p 511-521.

5. Chen S. J., Hwang C. L., Hwang F. P. Fuzzy multiple attribute decision making- methods and applications. Lecture notes in economics and mathematical systems. New York: Springer Verlag, 1992. $531 \mathrm{p}$.

6. Clemen R.T. Naturalistic decision making and decision analysis. Journal of behavioral decision making. 2001, Vol 14 (5), p 359-361.

7. Magyla T., Švėgžda O. The Methodology of Group Expert Evaluation. In: Proceedings of 8-th annual International Scientific and Technical Conference "RADIO-ELECTRONICS, ELECTRICAL and POWER ENGINEERING 2002": Conference proceedings. Moscow, 2002, p 282-287.

8. Dubois D., Land J., Prade H. Automated reasoning using probabilistic logic-semantics, belief revision, and variable certainty weights. IEEE transactions on knowledge and data engineering. 1994, Vol 6, p 64-71. 
9. Walley P. Measures of uncertainty in expent systems. Artificial intelligence. 1996, Vol 83 (1), p l-58.

10. Magyla T. Evaluation of Ebilock 950 Interlocking System Implementation by Using Analytic Hierarchy Process. Transport engineering (TRANSPORTAS). Vilnius: Technika, 2001, Vol XVI, No 5, p 131-137 (in Lithuanian).

11. Finan J. S., Hurley W. J. The analytic hierarchy process: does adjusting a pair-wise comparison matrix to improve the consistency ratio help? Computers \& Operations Research. 1997, Vol 24, p 749-755.

12. Kirkwood C. W., Corner J. L. The effectiveness of partial information about attribute weights for ranking alternatives in multi-attribute decision-making. Organizational behavior and human decision processes, 1993, Vol 54 (3), p 456476.

13. Magyla T. Building an expert system for railways automation. Electronics and electrotechnics. Kaunas: Technology, 2001, No 4 (33), p 68-71.

14. Campos L. M., Huete J. F. Independence concepts in possibility theory. Fuzzy sets and systems, 1999, Vol 103, p 127152.

15. Dias O. P. Ranking altematives using fuzzy numbers- a com- putational approach. Fuzzy sets and systems, 1993, Vol 56, p 247-252.

16. Teng J. Y., Tzeng G. H. Transportation investment project selection using fuzzy multi-objective programming. Fuzzy; sets and systems, 1998, Vol 96, p 259-280.

17. Legarth J. B. Environmental Decision Making for Recycling Options. $R \& C$ and Recycling, 1997, Vol 17, p 109-135.

18. Nayara M. Characterization of measures based on strict triangular norms. Journal of mathematical analysis and applications, 1999, Vol $236(2)$, p 370-383.

19. Henrion M., Breese J. S., Horvitz E. J. Decision-analysis and expert systems. AI magazine, 1991, Vol 12, p 64-91.

20. Chen C. B., Klein C. M. A simple approach to ranking a group of aggregated fuzzy utilities. IEEE transactions on systems man and cybernetics. Part B-Cybernetics, 1997, Vol 27, p 26-35.

21. Ralescu D. A., Sugeno M. Fuzzy integral representation. Fuzz)' sets and systems, 1996, Vol 84 (2), p 127-133.

22. Dubois D., Marichal J. L., Prade H., Roubens M., Sabbadin $\mathrm{R}$. The use of the discrete Sugeno integral in decision-making: A survey. International journal of uncertainty fuzziness and knowledge-based systems, 2001, Vol 9, p 539-561. 